\title{
Detection of Branch Retinal Vein Occlusion using Fractal Analysis
}

\author{
Juil J. Zode \\ Department of Electronics and \\ Telecommunication \\ Fr. C. Rodrigues Institute of Technology \\ Navi Mumbai, India
}

\author{
Pranali C. Choudhari \\ Department of Electronics and \\ Telecommunication $\mathrm{Fr}$. \\ C. Rodrigues Institute of Technology \\ Navi Mumbai, India
}

\begin{abstract}
Human retina consists of repeating pattern of arteries and veins. This repeating pattern is known as Fractals. Fractal Dimension is the quantity which is to be determined for analyzing disorder in retina using fractal analysis. There are two disorders of retina which leads to complete vision loss. They are Occlusion and Diabetic Retinopathy. Occlusion is due to arteries crossing over vein leading to bursting of vein which causes hemorrhage. There are three types of occlusions. They are Central Retinal Vein Occlusion (CRVO), Branch Retinal Vein Occlusion (BRVO) and Hemi-Retinal Vein Occlusion (HRVO). CRVO affects the center vein of retina, BRVO occurs at the branch of vein and Hemi-retinal affects the sub veins of main vein in retina. Out of these occlusions, Branch Retinal Vein Occlusion (BRVO) occurs most commonly. Diabetic Retinopathy is seen in diabetes patients. Their fractal dimension is different than that of occlusion patients. The paper concentrates on using fractal analysis for detection of Branch Retinal Vein Occlusion. The methods to determine fractal dimensions are Box Counting Method, Mass-Radius Method and Density-Density Correlation Method. Out of which Box Counting and Mass-Radius Method gives more accurate results.
\end{abstract}

\section{General Terms}

Fractal, fractal analysis, retinal vein occlusion.

\section{Keywords}

Diabetic retinopathy; occlusion; box counting method; massradius method

\section{INTRODUCTION}

Diabetic Retinopathy and Occlusions are the two disorders which can lead to vision loss. Diabetic Retinopathy is a diabetes complication that affects retina. It affects the blood vessel of the light-sensitive tissue at the back of the retina. This damage to blood vessels can lead to blindness. Occlusion is the blockage or closing of a blood vessel. Retinal Vein Occlusion (RVO) is a common disorder of the retina and one of the most common causes of vision loss worldwide. There are three types of occlusions. They are Central Retinal Vein Occlusion (CRVO), Branch Retinal Vein Occlusion (BRVO) and Hemi-Retinal Vein Occlusion (HRVO). CRVO is occlusion at the central retinal vein, BRVO is occlusion at branch of retinal vein and HRVO is occlusion at primary superior branch or primary inferior branch involving half of the retina. Retinal Vein Occlusion (RVO) is further divided into nonischemic and ischemic types. Ischemic is restriction in blood supply to tissues, which results in shortage of oxygen and glucose needed for cellular metabolism. Ischemia is generally because of problem with blood vessels with resultant damage to or dysfunction of tissue. Almost $2 / 3^{\text {rd }}$ of ischemic patients develop dreaded complications of macular edema, macular ischemia and neovascularization that leads to blindness. This paper concentrates on use of fractal analysis for detection of Branch Retinal Vein Occlusion. Fractal analysis is assessing characteristics of fractals. Fractals are the repeating pattern seen in retina made up of arteries and veins. Human retina is made up of fractals and analyzing those would help in determining disorder in retina. For analysis purpose fractal dimension has to be calculated. There are three methods to determine fractal dimension. They are Box Counting Method, Mass-Radius Method and Density-Density Correlation Method. Out of the three occlusions, Branch Retinal Vein Occlusion is the most common type of the occlusion occurring. To determine Branch Retinal Vein Occlusions, the above mentioned three methods are used. Out of those methods, Box Counting and Mass-Radius Methods gives accuracy in results.

\section{LITERATURE}

For Fractal analysis of retina, fractal dimension has to be calculated. Evaluating fractal characteristics would result in fractal analysis. A advance approach to study vascular systems is using fractal analysis to characterize the blood vessels. The first step is to determine if the vascular pattern could be characterize as fractals and then to measure fractal dimension. Fractal dimension is a ratio of how pattern changes with the scale at which it is measured. It is also said as a number that characterizes the distribution of the branching vascular system in 2-dimensional space. Natural structures have a similar appearance when viewed at different magnifications. When the basic pattern is magnified, one can observe repeating level of details each level looks like the whole. Rivers, mountains are some examples. Biological patterns also obeys this scaling relations; For example, trees are self-similar objects. If focused on smaller portion it resembles whole tree. This example tells about the concept of self-similar. A self-similar object has structure on all length scales and every part is same as the whole. It was Mandelbrot who explored the applications of fractal concepts to describe complex natural shapes, forms, and patterns. It was he who elucidated $\mathrm{D}$ as dimension and named it as fractal dimension. Fractal geometry concept was also found out by him. Fractal geometry is used to describe complex shapes, such as clouds or mountains. Fractal objects are different from Euclidean objects as for euclidean objects the mass of the object scales with a length raised to an integral power. Fractal objects obeys mass-length scaling relationship however, the exponent (the fractal dimension, D) is not equal to the Euclidean dimension d. The exponent is, in general, nonintegral and less than the Euclidean dimension.

$$
\mathrm{M}(\mathrm{r}) \approx \mathrm{r}^{+\mathrm{D}}
$$


Where D equals fractal Dimension. Equation (1) applies to fractals when they are self-similar. In general, the fractal dimension $\mathrm{D}<\mathrm{d}$, where $\mathrm{d}$ equals the Euclidean dimension of the space in which the fractal is embedded. Fractal dimension values is usually a noninteger value. Also in addition to fractal dimension, property of lacunarity is useful for the study of blood vessels. Fractal dimension would be of great help as it can be also used as a clinical tool. Various retinal pathologies can be analyzed using fractal geometry.

There is no fundamental equation that governs fractal geometry. Fractal dimension is being obtained by boxcounting method. The fractal dimension for the right and left eye for the normal patient is same. The fractal dimension obtained for the normal retina using box-counting method is 1.6 is been stated in [1]. Fractal is defined as complex geometry of the objects in nature [2]. Human retina is made up of geometrical multifractals that means the pattern in which the fractals are repeating need not be same. The STARE database of forty retinal images have been used for determining fractal dimension [2]. The database has been analyzed for normal retina as well as pathological states of retina and in all retinal images multifractal behavior is also observed. Also it had been found out that box counting (capacity dimension ) gives more accurate results than Massradius. Density-Density Correlation Method have the least accuracy when compared to these two methods. To diagnose retinal disease, the nature of retinal arteries and veins plays a key role [3]. Fractal dimension can differentiate between normal and diabetic patients. The box-counting method has been used for calculating fractal dimension. Retinal Nerve Fibre (RNF) layer photographs and Fundus Fluoroscein angiogram (FFA) were used [3]. There is high level of linearity seen. Here, also Fractal dimension is calculated using box-counting method [4]. The paper also states that fractal dimension does not depend on the age or gender of the patient. In [4], the grid method was considered as more accurate method compared to mass-radius and density-density correlation method. The diseases like diabetic retinopathy, proliferative retinopathy, occlusions will have different fractal dimension. Martin A. Mainster states that the fractal dimension of retinal arteries is 1.63 and that of retinal veins is 1.71. Fractal geometry offers proper description of ocular anatomy. Fractals are branching pattern in retina. This branching pattern have two important fractal properties. One is self similarity that is small parts of the pattern exhibit the pattern's overall structure and second is decreasing density that is density decreases as it grows in size. The method used is mass-radius method. It has been noted that vascular density decreases from central to peripheral retina. Also it had been found out that density of artery drops faster than that of capillary and veins. That means retina is spanned least completely by arteries and most completely by capillaries [5]. The two important fractal features introduced are using appropriate image processing algorithm and measure of lacunarity which describes the characteristics of fractals that have same fractal dimension but different appearances. Fractal dimension is calculated using box-counting method [6]. It has been found out from the study that the fractal dimension of diabetic patients is greater than that of a normal human retina. Also image resolution has influence on fractal dimension has been noted [6], therefore resolution needs to be proper. Fractal analysis is used as its key feature of self-similarity describes the geometric pattern that remains constant. It is used to differentiate optic disc from other large and bright regions in retinal images [7]. The fractal dimension is higher in case of hard exudates, that are deposits developed after leakage from retinal capillaries. Box-counting method is being used to calculate fractal dimension [7]. Fourier fractal dimension has advantage of computing the fractal dimension of gray scale images and eliminates the need for image segmentation. In [8] also box-counting method is used to calculate fractal dimension. Box-counting requires segmentation of the vessels in the retinal images. Method to measure fractal dimension of retina images includes preprocessing to improve its quality using wavelet transforms and also Fourier Fractal dimension (FFD) to obtain fractal dimension of image. The step includes generation of fourier transform of the enhanced image. FFD is very less sensitive to noise as it does not require the image to be binarized. Rangaraj M. Rangayyan [9], focuses on detecting and classifying neovascularization caused by proliferative diabetic retinopathy in retinal fundus images. Here also, box-counting approach is being used for calculating fractal dimension and it has been found out that fractal dimension of diabetic patients is much higher than normal retina patients. Daxer proposed density-density correlation method for calculating fractal dimension [10].

Occlusion means obstruction. Vein occlusion means obstruction of blood flow in vein. There are three types of vein occlusions - Central retinal Vein occlusion (CRVO), Branch retinal vein occlusion (BRVO) and Hemi-Retinal Vein Occlusion (HRVO). Normal features of arteriovenous crossings are the proximity of thin walled vein and thick walled artery, the narrowing of venous lumen and the sharing by artery and vein of common adventitial sheath.This provide the setting for occlusion. The comparison of number of arteriovenous crossings in patients with and without Branch Retinal Vein Occlusions to determine whether arteriovenous crossings are common in patients with BRVO is seen [12]. Figure 1 shows vein is obstructed by an artery and hence hemorrhage has taken place.

i) Box Counting Method

Binary image of retina is covered with square boxes of length $\mathrm{L}$

\begin{tabular}{|c|}
\hline No. of boxes of side length are counted and denoted as \\
\hline $\begin{array}{c}\text { Decrement the size of the box from retinal image size } \\
\text { to the smallest pixel and repeat the procedure. }\end{array}$ \\
\hline Log-Log plot of $\mathrm{N}(\mathrm{L}) \mathrm{v} / \mathrm{s}$ side of box is plotted \\
\hline Slope D of above plot obtained corresponds to fractal \\
dimension
\end{tabular}




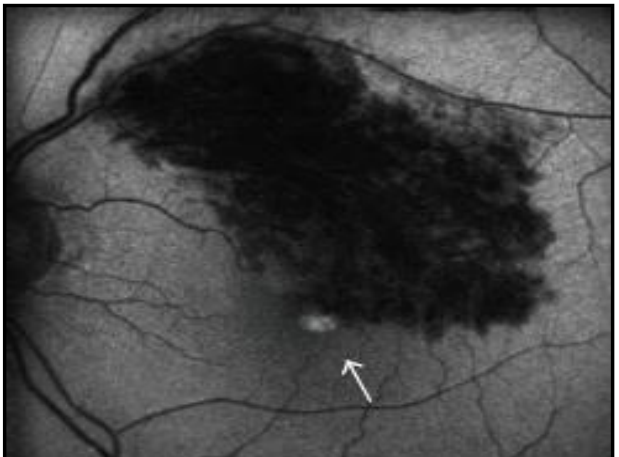

Figure 1: Branch Retinal Vein Occlusion [1]

ii) Mass-Radius Method

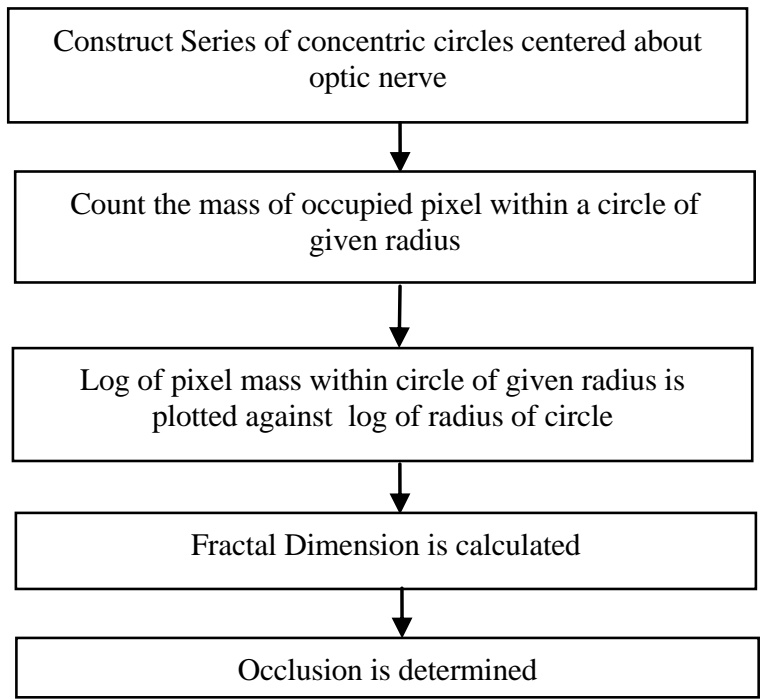

It has been found that Mean Platelet Volume (MPV) is higher in patients with Retinal Vein occlusions. MPV is the indicator of size and activity of platelets. Also it has been studied that MPV is elevated in occlusion diseases and hence MPV can be used as predictive tool for identifying risk for retinal vein occlusion patients. Higher level of mean platelet volume is risk factor for patients with occlusions is been presented [13]. Macular edema is cause of vision disturbance. It has been found that Optical Computed Tomography (OCT) and Fundus Autofluoroscence (FAF) are the two imaging techniques used in examining occlusion. FAF is a new technology used for characterizing retinal diseases. But OCT findings with respect to Branch Retinal Vein Occlusion and macular edema shows uniform autofluoroscence in eyes. Fig 2 shows the images obtained by OCT and autofluoroscence imaging. OCT makes note of morphological changes in the retina caused by occlusion while FAF is new technology that is used to characterize eyes with macular diseases [14]. The combination of these two techniques would provide more beneficial information about macular edema occurred due to branch retinal vein occlusion. Ultra Wide Field Fluoroscein Angiography (UWFFA) is a retinal imaging device used for capturing peripheral retinal findings [15]. OCT imaging is used for examining occlusions. OCT parameters like central macular thickness (CMT) and average retinal thickness is calculated of affected retina is stated [16]. In [19], the paper aims at increasing the possibility of optic disc detection in poor quality retinal images with exudates.

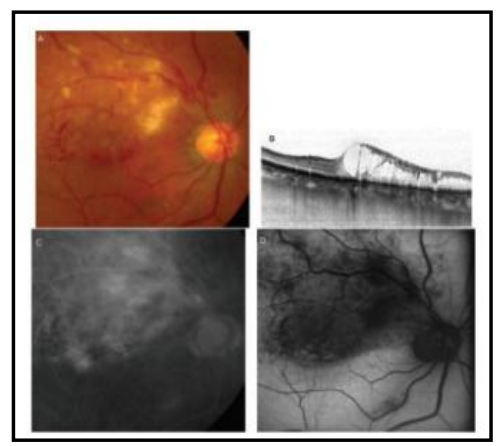

Figure 2: Fundus Photograph, OCT, Fluoroscein Angiograph and AutoFluoroscence images. [13]

Flowchart for fractal analysis of retinal images

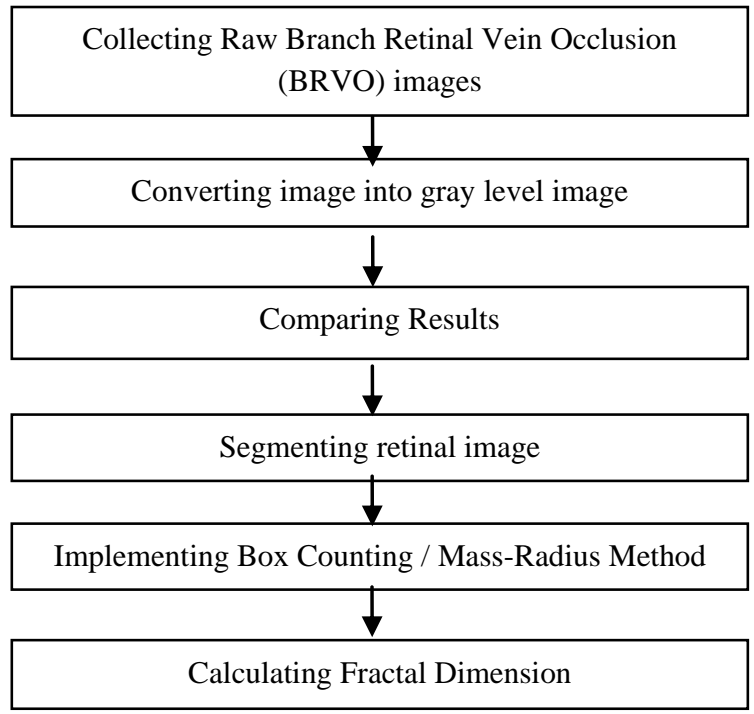

\section{CONCLUSION}

Diabetic Retinopathy and occlusion are the two disorders which lead to complete vision loss. Fractal analysis helps in detecting the occlusion. Fractal analysis can be done by three methods namely Box-counting, mass-radius and densitydensity correlation method. Out of which box-counting and mass-radius gives more accuracy. There are two imaging techniques which helps in detecting occlusions, they are Optical Computed Tomography (OCT) and Fundus AutoFluoroscence (FAF). The fractal dimension for normal retina is 1.6 and that of diabetic retinopathy patients is 1.71 calculated using mass-radius and box-counting method. The study on fractal analysis and detailed study on fractals and occlusions has been reviewed in this paper. Table 1 summarizes the work done in fractal analysis related to retina. Also it has been found out that the OCT images shows exact location of the occlusion and also tells how much is the depth of the occlusion. Hence it could be said that Fractal Analysis is promising analysis technique for detection of branch retinal vein occlusion, which is the most common type of occlusion occurring using fractal dimension. 
Table 1 Summary

\begin{tabular}{|c|c|c|c|c|}
\hline Author & Year & $\begin{array}{l}\text { Sample } \\
\text { Size }\end{array}$ & Method used & Observation \\
\hline Tatijana Stosic et al [2] & 2006 & 20 & Box-Counting & $\begin{array}{l}\text { Retina is made up of multifractals and fractal dimension is } \\
\text { calculated for normal retina as } 1.6 \text {. }\end{array}$ \\
\hline Gabriel Landini et al [4] & 1993 & 23 & Box-Counting & $\begin{array}{l}\text { Fractal analysis has been done on retina and box-counting output } \\
\text { has been considered more appropriate than mass-radius output. Also } \\
\text { its has been found out that fractal dimension does not change with } \\
\text { age/gender. }\end{array}$ \\
\hline Martin A. Mainster [5] & 1990 & 10 & Mass-Radius & $\begin{array}{l}\text { Fractal geometry gives accurate description of ocular anatomy and } \\
\text { fractal dimension for diabetic retinopathy is higher than that of } \\
\text { normal retina. The fractal dimension of diabetic retinopathy patients } \\
\text { is found to be } 1.71 \text {. }\end{array}$ \\
\hline Shu-Chen Cheng et al[6] & 2003 & 92 & Box-Counting & Image resolution has great influence on fractal dimension. \\
\hline M.Z. Azemim et al[8] & 2011 & 380 & Box-Counting & Retinal segmentation is essential for calculating fractal dimension \\
\hline $\begin{array}{l}\text { Sathvika Mudigonda et al } \\
\text { [9] }\end{array}$ & 2015 & NA & Box-Counting & $\begin{array}{l}\text { Retinal fundus images of diabetic patients is being studied. } \\
\text { Neovascularization due to diabetes increases the fractal dimension } \\
\text { to } 1.66 \text { from } 1.52 \text { of normal patients.. }\end{array}$ \\
\hline Irena Tsui et al[15] & 2013 & 84 & NA & $\begin{array}{l}\text { Out of the } 84 \text { patients, } 71 \text { patients had BRVO in superotemporal, } \\
\text { inferotemporal and superonasal quadrants. }\end{array}$ \\
\hline Loretta Ichim et al [19] & 2015 & 100 & Box-Counting & $\begin{array}{l}\text { Box-Counting is more efficient method for optic disc detection and } \\
\text { blood vessel segmentation is also very important. }\end{array}$ \\
\hline
\end{tabular}

\section{REFERENCES}

[1] Barry R. Masters, "Fractal Analysis of Normal Retinal Blood Vessels", Fractals, Vol. 2 No. 1, 103-110, 1994.

[2] Tatijana Stosic and Borko D. Stosic, "Multifractal Analysis of Human Retinal Vessels", IEEE transactions on medical imaging, Vol. 25 No.8, 1101-1107, 2006.

[3] V. Lakshminanarayanan, A.Raghuram, J.W.Myerson \& S. Varadharajan, "Fractal dimension of Retinal pathology ", Journal of Modern Optics, 2003.

[4] Gabriel Landini, Gary P. Misson and Philip I.Murray, "Fractal analysis of the normal human Retinal Fluoroscein Angiogram", Current Eye Research, Volume 12, No. 1, 23-27, 1993.

[5] Martin A. Mainster, "The Fractal Properties of Retinal Vessels: Embryological and Clinical Implications ", Eye, 235-241, 1990.

[6] Shu-Chen Cheng and Yueh-Min Huang, "A Novel Approach to Diagnose Diabetes Based on the Fractal Characteristics of Retinal Images", IEEE Transactions on Information Technology in Biomedicine, Vol. 7, No. 3, 163-170, 2003.

[7] Huajun Ying,Ming Zhang, Jyh-Charn Liu, "Fractal-based Automatic Localization and Segmentation of Optic Disc in Retinal Images" , Proceedings of the $29^{\text {th }}$ Annual International , 4139-4141, 2007.

[8] M.Z.Che Azemim, D.K.Kumar,T.Y.Wong, "Robust Methodology for Fractal Analysis of the Retinal Vasculature", IEEE Transactions on Medical Imaging, Vol. 30, No. 2, 243-250, 2010.

[9] Sathvika Mudigonda, Faraz Oloumi, Kalyana M. Katta and Rangaraj M. Rangayyan, "Fractal Analysis of
Neovascularization due to Diabetic Retinopathy in Retinal Fundus Images", The $5^{\text {th }}$ IEEE International Conference on E-Health and Bioengineering , 19-21, 2015.

[10] Albert Daxer, "The fractal geometry of proliferative diabetic retinopathy : implications for the diagnosis and the process of retinal vasculogenesis" , Volume 12, Number 12, 1103-1109, 1993.

[11] J. Jan, J. Odstrcilik, J. Gazerek, R.Kolar , "Retinal image analysis aimed at blood vessel tree segmentation and early detection of neural -layer deterioration", Computerized Medical Imaging and Graphics 36, Elseveir, 431-441, 2012.

[12] Jialiang Zhao, Srinivas M. Sastry , "Arteriovenous Crossing Patterns in Branch Retinal Vein Occlusions", 1992.

[13] Alparslan Sahin, Muhammed Sahin, Harun Yuksel, Fatih Mehmet Turkcu, Yasin Cinar, Abdullah Kursat Cingu, "The Mean Platelet Volume in Patients with Retinal Vein Occlusion", Hindawi Publishing Corporation, Journal of Ophthalmology, 2013.

[14] Tetsuju Sekiryu,Tomohiru Iida, Eiichi Sakai, Ichiro Maruko , "Fundus Autofluorescence and Optical Coherence Tomography Findings in Branch Retinal Vein Occlusion", Hindawi Publishing Corporation, Journal of Ophthalmology, 2012.

[15] Irena Tsui, Asima Bajwa, Valentina Franco-Cardenas, Carolyn K. Pan, Hanna Y. Kim and Stevan D.Schwartz, "Peripheral Fluoroscein Angiographic Findings in Fellow Eyes of Patients with Branch Retinal Vein Occlusion", Hindawi Publishing Corporation, International Journal of Inflammation, 2013. 
[16] Tatsuya Yunoki, Keiichi Mitarai,Shuichiro Yanagisawa, Tsuyoshi Kato, Nobuo Ishida, "Effects of Vitrectomy on Recurrent Macular Edema due to Branch Retinal Vein Occlusion after Intravitreal Injection of Bevacizumab", Hindawi Publishing Corporation, Journal of Ophthalmology, 2013.

[17] Seong Joon Ahn, Jeeyun Ahn, Se Joon Woo, Kyu Hyung Park , "Initial Dose of three Monthly Intravitreal Injections versus PRN intravitreal Injections of Bevacizumab for Macular Edema Secondary to Branch Retinal Vein Occlusion", Biomedical Research International, Hindawi Publishing corporation, 2013.
[18] Avnish Deobhakta and Louis K. Chang, "Inflammation in Retinal Vein Occlusion", International Journal of Inflammation, Hindawi Publishing Corporation, 2013.

[19] Loretta Ichim, Dan Popescu, Stefan Cirneanu "Combining Blood vessel Segmentation and Texture Analysis to improve Optic Disc Detection", The $5^{\text {th }}$ IEEE International Conference on E-Health and Bioengineering, 2015.

[20] Azemin M.Z, Fadilah Ab Hamid, "Box-Counting Fractal Dimension Algorithm Variations on Retina Images", Springer International Publishing, 337-343, 2016.

[21] Sveinn Hakon Hardarson, Einar Stefannsson, "Oxygen Saturation in Branch Retinal Vein Occlusion", Acta Ophthalmologica, 2011. 